\title{
BK Equation and Traveling Wave Solutions
}

\author{
J. T. de Santana Amaral ${ }^{1}$, M. A. Betemps ${ }^{1}$, M. B. Gay Ducati ${ }^{1}$, and G. Soyez ${ }^{2}{ }^{*}$ \\ ${ }^{1}$ Instituto de Física, Universidade Federal do Rio Grande do Sul, 91501 Porto Alegre (RS), Brazil \\ ${ }^{2}$ Service de Physique Theorique de Saclay, CEA/DSM/SPhT, F-91191 Gif-sur-Yvette, France
}

Received on 1st December, 2006

\begin{abstract}
It has been shown that the transition to the saturation regime of high energy QCD is similar to the formation of the front of a traveling wave. In particular, it can be verified that Balitsky-Kovchegov (BK) evolution equation reduces, after some approximations, to the nonlinear Fisher and Kolmogorov-Petrovsky-Piscounov (FKPP) equation, well-known from statistical physics. In these proceedings, based on the current knowledge of the asymptotic solutions of the BK equation, we propose a parametrization for the forward scattering amplitude which interpolates between the traveling wave solution and the saturation region.
\end{abstract}

Keywords: Traveling waves; Transition to saturation

\section{INTRODUCTION}

Solving the problem of the growth of the cross sections for hadronic interactions with energy is still an important challenge in Quantum Chromodynamics (QCD). As well-known, the increase of energy causes a fast growth of the gluon density and consequently of the cross sections. At very high energies, it is expected that, at some point, gluon recombination and multiple scattering might be important and unitarity can be restored. This phenomenon is called saturation and has been deeply investigated over the last thirty years [1-6].

From the theoretical side, the main contribution to describe and understand the saturation in high energy QCD comes from the development of non-linear QCD equations describing the evolution of scattering amplitudes towards this limit. The simplest of such equations is the Balitsky-Kovchegov (BK) equation $[7,8]$, which corresponds to the Balitsky-Fadin-KuraevLipatov (BFKL) [9] linear evolution equation with the addition of a non-linear term responsible for the tame of the growth of gluon density.

From the phenomenological side one has the discovery of geometric scaling in deep inelastic scattering (DIS) at HERA [10]. This phenomenological feature of high energy DIS is expressed as a scaling property of the virtual photon-proton cross section

$$
\sigma^{\gamma^{*}-p}(Y, Q)=\sigma^{\gamma^{*} p}\left(\frac{Q^{2}}{Q_{s}^{2}(Y)}\right),
$$

that is, the cross section depends on the scaling variable $\tau=$ $Q^{2} / Q_{s}^{2}(Y)$ instead of $Q^{2}$ and $Q_{s}^{2}(Y)$ separately. Here $Q$ is the virtuality of the photon, $Y$ is the total rapidity $(Y=\log (1 / x))$ and $Q_{s}(Y)$ is an increasing function of $Y$ called saturation scale. Indeed, geometric scaling has a natural explanation [11] in terms of traveling wave solutions of the BK equation.

In these proceedings we use the dipole model [12] to relate the $\gamma^{*} p$ cross section to the dipole-proton forward scattering

* On leave from the fundamental theoretical physics group of the University of Liège amplitude through a parametrization for the latter in momentum space. In Section II we relate $\sigma^{\gamma^{*} p}$ to the dipole-proton scattering amplitude through the dipole framework. In Section III we introduce the BK equation which describes the dipole scattering amplitude and the properties of its solutions, and from these we build the parametrization for the amplitude in Section IV. In Section V we present the conclusions and discuss the applications of our model.

\section{MUELLER'S DIPOLE PICTURE}

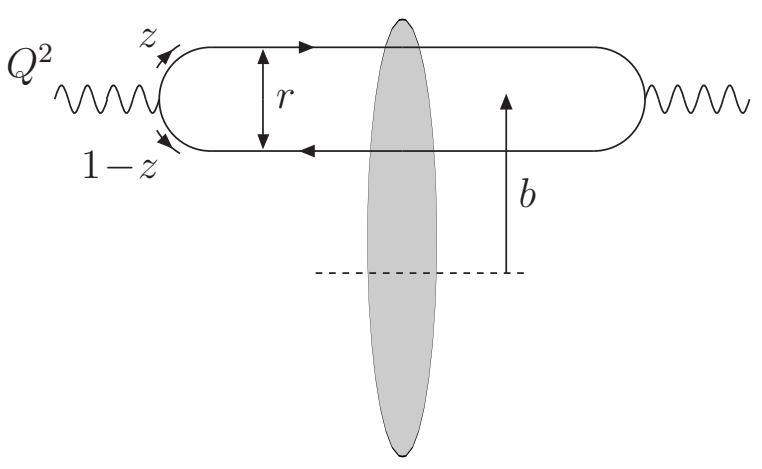

FIG. 1: Picture of the dipole model.

Let us consider the collision between a virtual photon and a proton at high energy. In a frame where the photon travels fast, but most of the energy is still carried by the proton, one can consider that the photon fluctuates into a $q \bar{q}$ (quark-antiquark) pair, a colorless dipole, which then interacts with the proton [12]. In the leading logarithmic approximation (LLA) of perturbative QCD (pQCD), the cross section factorizes and one gets the well-known formula

$$
\sigma^{\gamma^{*} p}(Y, Q)=\int_{0}^{\infty} r d r \int_{0}^{1} d z|\Psi(z, r, Q)|^{2} \sigma_{d i p}^{\gamma^{*} p}(r, Y),
$$

where $r$ is the size of the dipole, $z$ is the longitudinal momentum fraction of the photon carried by the quark and $\sigma_{d i p}^{\gamma^{*} p}(r, Y)$ 
is the dipole-proton cross section. Assuming an independence on the impact parameter of the collision, this cross section is proportional to the proton-dipole forward scattering amplitude, $T(r, Y)$, through the relation

$$
\sigma_{\text {dip }}^{\gamma^{*} p}(r, Y)=2 \pi R_{p}^{2} T(r, Y),
$$

where $R_{p}^{2}$ is the proton radius. As we shall see in the next section, the amplitude $T(r, Y)$ obeys the BK equation in coordinate space but the asymptotic behaviour of its solutions is naturally expressed in momentum space. We want to express $\sigma^{\gamma^{*} p}$ in terms of $\tilde{T}(k, Y)$, the Fourier transform of $T(r, Y)$ :

$$
T(k, Y)=\frac{1}{2 \pi} \int \frac{d^{2} r}{r^{2}} e^{i \mathbf{k} \cdot \mathbf{r}} T(r, Y)=\int_{0}^{\infty} \frac{d r}{r} J_{0}(k r) T(r, Y) .
$$

\section{SCATTERING AMPLITUDES AT HIGH-ENERGY}

Consider now a fast-moving colorless $q \bar{q}$ dipole of transverse size $r=|\mathbf{x}-\mathbf{y}|$, where $\mathbf{x}$ and $\mathbf{y}$ are the coordinates of the quark and antiquark, respectively, interacting with a given dense target. In the large- $N_{c}$ approximation $\left(N_{c}\right.$ is the number of colors), and in the mean-field approximation, the highenergy behaviour of the dipole forward scattering amplitude $T(\mathbf{x}, \mathbf{y} ; Y)$ follows the BK equation [7, 8]. In coordinate space this equation reads

$$
\begin{gathered}
\partial_{Y} T(\mathbf{x}, \mathbf{y} ; Y)=\frac{\bar{\alpha}}{2 \pi} \int d^{2} \mathbf{z} \frac{(\mathbf{x}-\mathbf{y})^{2}}{(\mathbf{x}-\mathbf{z})^{2}(\mathbf{z}-\mathbf{y})^{2}} \\
{[T(\mathbf{x}, \mathbf{z} ; Y)+T(\mathbf{z}, \mathbf{y} ; Y)-T(\mathbf{x}, \mathbf{y} ; Y)-T(\mathbf{x}, \mathbf{z} ; Y) T(\mathbf{z}, \mathbf{y} ; Y)]}
\end{gathered}
$$

which, as it was said in the Introduction, corresponds to the BFKL equation (the first three terms inside the brackets), but with an additional term (the term quadratic in $T$ ) which by its turn corresponds to the nonlinear effects responsible for the decreasing of the growth in the gluon density. Here $\bar{\alpha}=\alpha_{s} N_{c} / \pi, \alpha_{s}$ is the strong coupling constant (considered fixed). If one neglects the dependence on the impact parameter $\mathbf{b}=(\mathbf{x}+\mathbf{y}) / 2$ and integrates out the remaining angular dependence of $\mathbf{r}$, Eq.(4) becomes an equation for $T(r, Y)$. The latter can be expressed in momentum space through (3).

The amplitude $\tilde{T}(k, Y)$ obeys the BK equation in momentum space

$$
\partial_{Y} \tilde{T}=\bar{\alpha} \chi\left(-\partial_{L}\right) \tilde{T}-\bar{\alpha} \tilde{T}^{2}, \quad \bar{\alpha}=\frac{\alpha_{s} N_{c}}{\pi},
$$

where $\alpha_{s}$ is the strong coupling constant, $N_{c}$ is the number of colours,

$$
\chi(\gamma)=2 \psi(1)-\psi(\gamma)-\psi(1-\gamma)
$$

is the characteristic function of the Balitsky-Fadin-KuraevLipatov (BFKL) kernel [9] and

$$
L=\log \left(\frac{k^{2}}{k_{0}^{2}}\right)
$$

where $k_{0}$ is some fixed low momentum scale. The kernel $\chi(\gamma)$ is an integro-differential operator which may be defined with the help of the formal series expansion

$$
\begin{aligned}
& \chi\left(-\partial_{L}\right)=\chi\left(\gamma_{0}\right) \mathbf{1}+\chi^{\prime}\left(\gamma_{0}\right)\left(-\partial_{L}-\gamma_{0} \mathbf{1}\right) \\
& +\frac{1}{2} \chi^{\prime \prime}\left(\gamma_{0}\right)\left(-\partial_{L}-\gamma_{0} \mathbf{1}\right)^{2}+\frac{1}{6} \chi^{(3)}\left(\gamma_{0}\right)\left(-\partial_{L}-\gamma_{0} \mathbf{1}\right)^{3}+\ldots
\end{aligned}
$$

for some $\gamma_{0}$ between 0 and 1, i.e. for the principal branch of the function $\chi$.

\section{A. Traveling wave solutions}

If one performs the following change of variables

$$
t \sim \bar{\alpha} Y, \quad x \sim \log \left(k^{2} / k_{0}^{2}\right), \quad u(x, t) \sim \tilde{T}(k, Y)
$$

it has been shown [11] that the BK equation reduces to the Fisher and Kolmogorov-Petrovsky-Piscounov (F-KPP) equation [13] for $u$ when its kernel (6) is approximated by the first three terms of the expansion, the so-called diffusive approximation. The F-KPP equation is a well-known equation in non-equilibrium statistical physics, whose dynamics is called reaction-diffusion dynamics, and it has the form

$$
\partial_{t} u(x, t)=\partial_{x}^{2} u(x, t)+u-u^{2},
$$

where $t$ is time and $x$ is the coordinate. This equation admits the so-called traveling wave solutions.

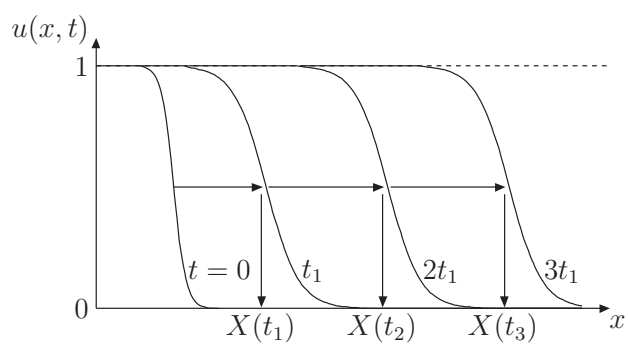

FIG. 2: Traveling wave behaviour for $u(x, t)$, solution of Eq.(9).

For a traveling wave solution one can define the position of a wave front $x(t)=v(t)$, irrespective of the details of the nonlinear effects. At larger times, the shape of a traveling wave is preserved during its propagation, and the solution becomes only a function of the scaling variable $x-v_{c} t$, where $v_{c}$ is the critical velocity.

In the language of saturation physics the position of the wave front is nothing but the saturation scale

$$
x(t) \sim \ln Q_{s}^{2}(Y)
$$

and the scaling cited above corresponds to the geometric scaling

$$
x-v_{c} t \sim \ln k^{2} / Q_{s}^{2}(Y)
$$


The linear part of (5) is solved by [11]

$$
\tilde{T}(k, Y)=\int \frac{d \gamma}{2 \pi i} \tilde{T}_{0}(\gamma) \exp (-\gamma L+\bar{\alpha} \chi(\gamma) Y)
$$

where $\tilde{T}_{0}(\gamma)$ is the Mellin transform of the initial condition at $Y=0$. The velocity of the front is given by

$$
v_{c}=\min _{\gamma} \bar{\alpha} \frac{\chi(\gamma)}{\gamma}=\bar{\alpha} \frac{\chi\left(\gamma_{c}\right)}{\gamma_{c}}=\bar{\alpha} \chi^{\prime}\left(\gamma_{c}\right)
$$

where $\gamma_{c}$ is the saddle point of the exponential phase factor. This fixes, for the BFKL kernel, $\gamma_{c}=0.6275 \ldots$, and $v_{c}=$ $4.88 \bar{\alpha}$.

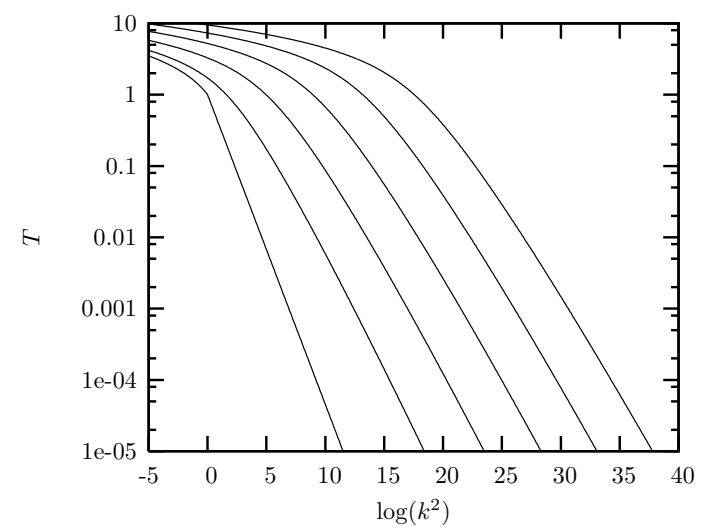

FIG. 3: Numerical solution to BK equation for increasing (from left to right) values of rapidity $Y=0,5,10,15,20,25$. The traveling wave behaviour with increasing rapidity is clearly manifest.

In terms of QCD variables, the dipole forward scattering amplitude in momentum space in the tail of the wave front reads

$\tilde{T}(k, Y) \stackrel{k \gg Q_{s}}{\approx}\left(\frac{k^{2}}{Q_{S}^{2}(Y)}\right)^{-\gamma_{c}} \log \left(\frac{k^{2}}{Q_{S}^{2}(Y)}\right) \exp \left[-\frac{\log ^{2}\left(k^{2} / Q_{s}^{2}(Y)\right)}{2 \bar{\alpha} \chi^{\prime \prime}\left(\gamma_{c}\right) Y}\right]$.

The saturation scale is defined as

$Q_{s}^{2}(Y)=k_{0}^{2} \exp \left(\bar{\alpha} v_{c} Y-\frac{3}{2 \gamma_{c}} \log (Y)-\frac{3}{\gamma_{c}^{2}} \sqrt{\frac{2 \pi}{\bar{\alpha} \chi^{\prime \prime}\left(\gamma_{c}\right)}} \frac{1}{\sqrt{Y}}\right)$.

\section{CONNECTION WITH SATURATION}

The goal of this work is to describe the matching around the saturation scale between two regions: the one described by the tail of the wave front, Eq.(14) and the other one, characterized by saturation. The latter can be parametrized as

$$
\tilde{T}(k, Y) \stackrel{k \ll Q_{s}}{\approx} c-\log \left(\frac{k}{Q_{s}(Y)}\right) \text {, }
$$

where $c$ is an arbitrary constant.

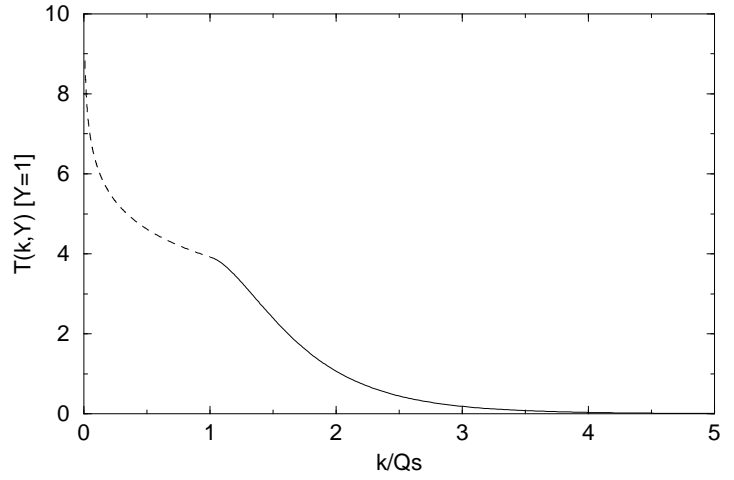

FIG. 4: Matching procedure between the tail of the wave front (solid line) and the saturation region (dashed line) through the continuity of $\tilde{T}(k, Y)$ and its first derivative for $Y=1$.

Given that the fully asymptotic behaviour of the amplitude is described by (14) and (16), our first attempt to connect both was to use both behaviours separately and match the constant $c$ from continuity conditions (see Figure 4).

However, such matching procedure does not necessarily imply a positive Fourier transform of the scattering amplitude. Then, the best way to obtain the description of such transition to the saturation region is to make an analytic interpolation procedure. The idea is to build the saturation domain from the dilute one and the starting point is a function, that we call $\tilde{T}_{\text {dil }}$, which reproduces (up to the logarithmic factor) the amplitude for diffusive scaling (14),

$$
\tilde{T}_{\text {dil }}=A \exp \left[-\gamma_{c} \log \left(\frac{k^{2}}{Q_{s}^{2}(Y)}\right)-\frac{L_{\text {red }}^{2}-\log ^{2}(2)}{2 \bar{\alpha} \chi^{\prime \prime}\left(\gamma_{c}\right) Y}\right]
$$

with

$$
L_{\text {red }}=\log \left[1+\frac{k^{2}}{Q_{s}^{2}(Y)}\right]
$$

and write $\tilde{T}$ in terms of it in such a way that we can recover the asymptotic behaviours of $\tilde{T}$, i.e., Eqs. (14) and (16).

One expression which satisfies these properties is the following:

$$
\tilde{T}(k, Y)=\frac{L_{F}}{1+\frac{1}{\tilde{T}_{\mathrm{dil}}}},
$$

where the logarithmic factor

$$
L_{F}=\sqrt{K^{2}+\frac{1}{4} \log ^{2} \frac{k^{2}}{Q_{s}^{2}(Y)}}
$$

The constants $A$ and $K$ can be determined by applying the parametrization proposed to describe some physical observable, for example, via fitting procedure to some experimental data. Such procedure could be a good way to test the robustness of our model. 


\section{CONCLUSIONS}

In this work we have investigated the matching between saturation and travelling wave solutions of the BK equation which describe the forward scattering amplitude at high energies in the virtual photon-proton scattering. As the result does not necessarily imply a positive Fourier transform of the amplitude, we concluded that the best way to connect both solutions is through an analytic interpolation model. An expression for the amplitude, Eqs.(17)-(20), has been proposed and tested in order to be used to describe some experimental data. This work is in progress.

\section{Acknowledgements}

This work is partially supported by CNPq, Brazil. G.S. is funded by the National Funds for Scientific Research (FNRS, Belgium).
[1] L. V. Gribov, E. M. Levin, and M. G. Ryskin, Phys. Rep. 100, 1 (1983).

[2] A. H. Mueller and J. Qiu, Nucl. Phys. B 268, 427 (1986).

[3] A. H. Mueller, Nucl. Phys. B 335, 115 (1990).

[4] J. Jalilian-Marian, A. Kovner, A. Leonidov, and H. Weigert, Nucl. Phys. B 504, 415 (1997) [arXiv:hep-ph/9701284]; E. Iancu, A. Leonidov, and L. D. McLerran, Nucl. Phys. A 692, 583 (2001) [arXiv:hep-ph/0011241]; H. Weigert, Nucl. Phys. A 703, 823 (2002) [arXiv:hep-ph/0004044].

[5] A. L. Ayala, M. B. Gay Ducati, and E. M. Levin, Nucl. Phys. B 493, 305 (1997); Nucl. Phys. B 551, 335 (1998).

[6] Yu. V. Kovchegov and A. H. Mueller, Nucl. Phys. B 529, 451 (1998).

[7] I. I. Balitsky, Nucl. Phys. B 463, 99 (1996); Phys. Rev. Lett. 81, 2024 (1998); Phys. Lett. B 518, 235 (2001); arXiv:hep$\mathrm{ph} / 0101042$.

[8] Y. V. Kovchegov, Phys. Rev. D 60, 034008 (1999); 61, 074018
(2000).

[9] L. N. Lipatov, Sov. J. Nucl. Phys. 23, 338 (1976); E. A. Kuraev, L. N. Lipatov, and V. S. Fadin, Sov. Phys. JETP 45, 199 (1977); I. I. Balitsky and L. N. Lipatov, Sov. J. Nucl. Phys. 28, 822 (1978).

[10] A. M. Stasto, K. Golec-Bernat, and J. Kwiecinski, Phys. Rev. Lett. 86, 596 (2001).

[11] S. Munier and R. Peschanski, Phys. Rev. Lett. 91, 232001 (2003); Phys. Rev. D 69, 034008 (2004); Phys. Rev. D 70, 077503 (2004).

[12] A. H. Mueller, Nucl. Phys. B 415, 373 (1994); A. H. Mueller and B. Patel, Nucl. Phys. B 425, 471 (1994); A. H. Mueller, Nucl. Phys. B 437, 107 (1995).

[13] R. A. Fisher, Ann. Eugenics 7, 355 (1937); A. Kolmogorov, I. Petrovsky, and N. Piscounov, Moscou Univ. Bull. Math. A 1, 1 (1937). 